\title{
Análisis de Política Pública de Infancia y Adolescencia, 2011-2021, en Bogotá, D. C., Colombia
}

\author{
NATHALy RoZo-GutiÉRreZ* \\ Zulma VARgas-TrujILLO**
}

Artículo recibido: 17 de enero de 2017

Artículo aprobado: 12 de diciembre de 2017

Doi: http://dx.doi.org/10.12804/revistas.urosario.edu.co/desafios/a.5325

Para citar este artículo: Rozo-Gutiérrez, N. \& Vargas Trujillo, Z. (2018). Análisis de la Política Pública de Infancia y Adolescencia, 2011-2021, en Bogotá, D. C., Colombia. Desafíos, 30(2), 279-314. Doi: http://dx.doi.org/10.12804/revistas.urosario.edu.co/desafios/a.5325

\section{Resumen}

Los derechos humanos, que incluyen los derechos de los niños, son concebidos como el contenido sustancial del sistema democrático y enmarcan al Estado y a la construcción social de las familias. El objetivo fue analizar el desarrollo y puesta en marcha de la política pública de la infancia y adolescencia 2011-2021 para Bogotá. Se realizó un análisis cualitativo de base documental fundamentado en la metodología propuesta por Ordoñez-Matamoros. Los resultados evidenciaron que las necesidades definidas fueron interpretadas a conveniencia del tomador de decisión y de acuerdo con la estructura de gobierno de la política. El proceso de identificación de tipologías de la política pública

\footnotetext{
* Magíster en Salud Pública. Línea de profundización de Políticas Públicas. Facultad de Medicina. Universidad El Bosque. Bogotá, D. C., Colombia. Correo electrónico: jrozog@ unbosque.edu.co. ORCID: http://orcid.org/0000-0002-1201-7681

** Magíster en Salud Pública. Línea de profundización de Políticas Públicas. Facultad de Medicina. Universidad El Bosque. Bogotá, D. C., Colombia. Correo electrónico: zulvar78@ yahoo.com. ORCID: http://orcid.org/0000-0001-9236-2436
} 
distrital permitió comprender la complejidad del ejercicio y entender que en muchos casos es válida la existencia implicita de múltiples tipos, debido a la diversidad de los contenidos e intencionalidades enmarcados en ella; sin embargo, es necesario que sea incluyente hacia la población en general para que asi se transforme en una ventana de oportunidades para el desarrollo bumano en todas sus dimensiones.

Palabras clave: políticas públicas, adolescente, prescolar, atención integral de salud, salud pública.

\title{
Analysis of the Public Policy Regarding Children and Adolescents, 2011-2021 in Bogotá, Colombia
}

\begin{abstract}
The human rights that integrate the rights of children are conceived of as the substantial content of a democratic system and consequently frame the state and the social construction of families. The objective of this article is the analysis of the development and implementation of the public policy for children and adolescents in 2011-2021 for the city of Bogotá. The methodology used is qualitative document analysis. In terms of the identification of the problem, it is shown that the policy-makers took the defined needs and the governance structure of the policy into account and accordingly presented several alternatives. The process of identifying typologies of public policy according to districts made it possible to understand the complexity of the exercise and that in many cases the implicit existence of multiple types is due to the diversity of contents and embedded intentions. However, it is necessary that the policy is inclusive towards the population in general so that it becomes a window of opportunity for human development in all of its dimensions.
\end{abstract}

Keywords: public policy, adolescents, comprehensive health care, public bealth. 


\title{
Análise da política pública de infância e adolescência 2011-2021 Bogotá, Colômbia
}

\begin{abstract}
Resumo
Os direitos bumanos que integram os direitos das crianças são concebidos como o conteúdo substancial do sistema democrático e enquadram ao Estado e a construção social das familias. O objetivo foi analisar o desenvolvimento e partida da política pública da infância e adolescência 2011-2021 para Bogotá. Se realizou uma análise qualitativa de base documental fundamentada na metodologia proposta por Ordoñez-Matamoros. Os resultados evidenciaram que as necessidades definidas foram tomadas a conveniência do tomador de decisão e de acordo com a estrutura de governo da politica. O processo de identificação de tipologias da política pública distrital permitiu compreender a complexidade do exercício e entender que em muitos casos é válido, a existência implícita de múltiplos tipos, devido à diversidade dos conteúdos e intencionalidade enquadrados nela, no entanto, é preciso que seja inclusiva à população em geral para que assim se transforme em uma janela de oportunidades para o desenvolvimento humano em todas suas dimensões.
\end{abstract}

Palavras-chave: politicas públicas, adolescente, pré-escolar, atenção integral de saúde, saúde pública.

\section{Introducción}

Este artículo pretende analizar el desarrollo y puesta en marcha de la Política Pública de Infancia y Adolescencia en Bogotá, D. C., 20112021, la cual fue producto de un compromiso por parte del gobierno distrital para mejorar y fortalecer el desarrollo de los niños, niñas y adolescentes. En primer lugar, se debe tener claro que la política es una red compleja de acciones compuesta por flujos de información interconectada bajo procesos de interacción en la cotidianidad del ser humano, con una base de poder y de justicia social (Skelton, 2013). Lo anterior permite la transformación de realidades, pensamientos y elecciones para la resolución de los problemas detectados por los individuos y los colectivos. 
Dichos problemas son categorizados como tales a partir de subjetividades expresadas por la población en escenarios públicos o privados de interacción, discusión y reflexión, donde se pueden clasificar como de interés común en las agendas gubernamentales y estatales. Es posible hablar de distintos tipos de agenda política. En la literatura, se distingue la agenda pública de la agenda formal para marcar dos momentos o escenarios que derivarán en distintas pautas de acción política. Se busca que los problemas logren la agenda formal que es aquella que se incorpora en las decisiones de gobierno y, para ello, suele haber un proceso desde la agenda pública, que es el debate cotidiano, en los escenarios sociales sobre un asunto que genera interés, inquietud o preocupación. Sobre la manera cómo pasan los temas de una agenda a otra se puede decir que los medios de comunicación son importantes, como lo son algunos grupos de interés que tienen relación con el asunto problemático (Cobb, Ross \& Ross, 1976).

De esta forma, la salud pública se convierte en un campo en el que confluyen tanto problemáticas como posibilidades de acción y es vista por los gobiernos como un escenario para responder a dichas situaciones territoriales de forma rápida y oportuna. Sin embargo, para que el sistema funcione, se requiere de un recurso humano que facilite lograr cobertura, acceso, disponibilidad y calidad. Este debe tener políticas robustas para mantener su incentivo y compromiso con las responsabilidades asignadas, así como promover una formación continua para el fortalecimiento de competencias traducidas en gestión interinstitucional, desarrollo de objetivos estratégicos y resultados en salud (Regan, MacDonald, Allan, Martin \& Peroff-Johnston, 2014).

Así es como la acción política se posiciona como una oportunidad real de concertar y construir algo desde la necesidad colectiva; esta trasciende la gestión por medio de una estructura de gobierno mediadora en la búsqueda cooperante de la resolución de los problemas a través de estrategias técnicas, operativas y comunicativas con el propósito de vincular al individuo de forma participativa y permanente (Payne, 2000). Esta intencionalidad mediadora y resolutiva transforma a "la política en nada más que la medicina a gran escala” (Duque Daza, 2013), porque 
se une en el esfuerzo de llevar a cabo acciones para mejorar la salud y modificar los problemas que afectan a la población (Milne, 2012).

A partir de ello, la política pública se constituye como un proceso de mediación social que posibilita la confrontación sectorial, para modificar las relaciones interinstitucionales, con el objeto de dar respuesta a las complejidades derivadas de la interacción humana (Muller, 2002). Como consecuencia de la transformación sectorial, en términos de dinámicas y procesos, se inicia el posicionamiento de las respuestas institucionales como acciones gubernamentales coordinadas desde la esfera político-administrativa (Roth, 2002).

Otro rasgo a tener en cuenta en el análisis de las políticas es el proceso multidisciplinar y multimetodológico que desarrolla, donde pretende "[...] explicar tanto las interacciones entre actores, instituciones, intereses e ideas como la naturaleza y el resultado de las decisiones y acciones que emergen, contribuyendo en el desarrollo de la misma a través del reconocimiento de fortalezas y debilidades" (Salas-Zapata, Ríos-Osorio, Gómez-Arias \& Castillo, 2012, p. 77; Howard, Gordon \& Jones, 2014), complementado con la monitorización de avances y visualización de la intencionalidad institucional para reconocer los actores sociales en una realidad que debe ser construida desde el territorio (Majone, 2005).

El niño, niña y adolescente tiene nociones conceptuales influenciadas por la historia y la cultura de cada contexto; las particularidades de crecimiento y desarrollo varían en cada grupo social; sin embargo, de forma genérica se consideran ciclos vitales aquellos donde ocurren los mayores desarrollos físicos, psicológicos y familiares con formas particulares de ver, sentir y observar el mundo. Para estas etapas de la vida, es esencial reconocer que se hace parte de ejes socializadores como la familia y el colegio, constituidos escenarios de interacción y aprendizaje para la construcción de la personalidad, dado que son entornos influyentes en la salud de la población (Carey, Crammond \& Keast, 2014).

Como consecuencia de sus continuos cambios físicos, psicológicos y sociales exigen políticas estatales dirigidas al cumplimiento de sus necesi- 
dades o interés bajo el marco del respeto y la protección de sus derechos en situaciones tales como participación en los conflictos armados, venta de niños, niñas y adolescentes, prostitución infantil y utilización en la pornografía. Así mismo, se deben gestionar marcos sociales y jurídicos en pro de la dignidad, la vida, la supervivencia, el bienestar, la salud, el desarrollo, la participación y la no discriminación, y que permitan la intervención y reducción de disparidades y sufrimientos inaceptables (Pautassu \& Royo, 2012).

En cuanto a que la infancia y la adolescencia sean categorías históricosociales, se hace fundamental el poder configurador del Estado y su institucionalidad como garantes de una política pública con respecto a las prioridades en el campo de la infancia. La primera condición para la defensa de los derechos de la infancia es la presencia de una institucionalidad estatal fuerte con competencias para lograr la igualdad; la presencia de un Estado como espacio político para luchar por un proyecto emancipatorio que comience por la infancia y la adolescencia (Butelo, 2012).

En este sentido, si existe una nula o baja autoridad estatal, los niños, niñas y adolescentes están expuestos a diversos factores que pueden alterar el crecimiento y el desarrollo esperado; entre estos, los nutricionales como el principal marcador fisiológico que proyecta la calidad de vida, los años de vida productivos, el proceso cognitivo que puede generarse en los menores de cinco años, así como la susceptibilidad a enfermedades transmisibles y no transmisibles (Nisbetta, Gillespieb, Haddadb \& Harrisb, 2014).

Se puede mencionar que algunos estudios hacen evidente que, para la intervención de los eventos prevalentes de cada territorio, se debe implementar un tipo de abordaje —o varios — que facilite una mirada de totalidad, de tal forma se pretende intervenir desde la comprensión de todos los elementos que lo integran (Tubbing, Harting \& Stronks, 2015). Un ejemplo de ello es el abordaje de la desnutrición o sobrepeso el cual ha sido netamente técnico y carece de una visión política nutricional amplia que permita desarrollar mecanismos económicos y acciones intersectoriales orientadas hacia la alimentación 
suficiente, oportuna y adecuada (Nisbetta, Gillespieb, Haddadb \& Harrisb, 2014).

Es así como las respuestas efectivas de salud se han construido desde varios ejes de acción: 1) la seguridad alimentaria en los hogares, relacionada con acceso, disponibilidad, cantidad e inocuidad de los alimentos; 2) prácticas de atención: lactancia materna, alimentación complementaria y prácticas preventivas; 3) construcción de un entorno más amplio en salud: acceso a agua potable, saneamiento básico adecuado y disponibilidad de un sistema de salud por medio de programas y servicios con acceso y cobertura universal. Otros incluyen la mitigación de la pobreza con el desarrollo de fuentes económicas tales como agricultura y otros medios de empleo que empoderan a la población y aumentan su poder adquisitivo.

De esta forma, el desarrollo económico, social y cultural se transforma en un vehículo para lograr la disminución de problemas demográficos y de desigualdades relacionadas con la baja oferta de empleo, la distribución desigual de ingreso y recursos, y la poca posibilidad de acceso a servicios y bienes. En este sentido, los propósitos de una política están orientados a 1) logro de un nivel de vida más alto; 2) la transformación de conductas individuales que se dan forma intergeneracional; 3) la participación de niños, niñas y adolescentes en las etapas de las políticas públicas; 4) la promoción de la respuestas institucionales ante el cambio demográfico y económico que se requiere para que los individuos se integren al desarrollo; y 5) la posibilidad de intervención de los niveles de pobreza, desarrollo y condiciones de vida para impactar en la morbilidad, la mortalidad, la migración, la fecundidad y la natalidad (Hernández, 2006; Argüello, 1991; Welti, s.f.; Valdés \& Ospina, 2011).

Para ello, las políticas, y todas sus perspectivas, se convierten en una estrategia que aborda el estancamiento económico, el alto crecimiento poblacional y los desenlaces fatales. Por consiguiente, es necesario que estas se planteen desde las necesidades de las personas, las familias y las comunidades porque así se podrían controlar consecuencias irreversibles de salud que se traducen en mecanismos de pobreza, y que 
son transmitidos de generación en generación (Nisbetta, Gillespieb, Haddadb \& Harrisb, 2014; Argüello, 1991).

Por esta razón el niño, niña y adolescente requiere de un trabajo interinstitucional e intersectorial con voluntad política y compromiso social para la garantía de los derechos de los niños, el desarrollo humano y de sus libertades, con la posibilidad del disfrute de su bienestar mitigando los factores de riesgo y eventos susceptibles que deterioran o retrasan el desarrollo individual y colectivo de un país, porque son ellos el futuro de las sociedades humanas (Nisbetta, Gillespieb, Haddadb \& Harrisb, 2014; Hernández, 2006; CEPAL, Naciones Unidas, UNICEF, SECIB, 2001).

De esta manera, la carga de la enfermedad y del deterioro de la calidad de vida en la población infantil y adolescente está relacionada con la morbimortalidad evitable y las discapacidades prevenibles que, desde una planificación multisectorial, como por ejemplo la política pública - que operacionaliza la gestión sectorial—, se pueden resolver o controlar. No obstante, al materializar la realidad del niño, niña y adolescente con la política de alto nivel, esta última se desconecta y se distancia de los intereses reales de los colectivos, por lo que se pierde la capacidad resolutiva, lo cual es un reto actual para los países y territorios: la adaptabilidad de las intervenciones a las percepciones locales de desarrollo, asegurando un marco de resultados exitosos en términos de efectividad y pertinencia (Nisbetta, Gillespieb, Haddadb \& Harrisb, 2014).

Es necesario, entonces, intervenir en la mejora de la política pública como mecanismo mediador y estratégico para el desarrollo humano y compuesta por recursos de tipo técnico (experiencias regionales), político (consenso político mínimo), estructurales (creación de condiciones productivas, sociales y culturales) y humano (equipos interdisciplinarios con una coordinación interinstitucional, herramientas para la gestión, participación de actores sociales, grupos de interés que contribuyan a la planeación, implementación y evaluación de la misma) bajo el precepto de que "el acceso al cuidado de la salud aumenta cuando existe una planificación dirigida por la comunidad" (Fox, 2002; Payne, 2000; Argüello, 1991). 
Por consiguiente, una de las barreras que se han identificado en los gobiernos de países en vías de desarrollo para el éxito de las políticas y del crecimiento social es la falta de respeto por la ley, la corrupción y la ineficacia de los tomadores de decisiones para proveer los recursos necesarios en términos de costo-beneficio, rentabilidad y calidad de los servicios (Sánchez, 2003). El rol de gobernanza de los Estados debe mediar las condiciones necesarias para que el ser humano desarrolle sus capacidades con libertad y autonomía, distanciándose de medidas gubernamentales paternalistas que encarecen y empobrecen al ser humano en su totalidad, convirtiéndolo en un ser pasivo, acrítico e irreflexivo de su contexto (Restrepo-Ochoa, 2013).

Se debe agregar que, para Colombia, la priorización del niño, niña y adolescente en las agendas de gobierno inicia en el año 2003 con varios espacios de debate, análisis y reflexión de la situación de la población caracterizada por la prevalencia de enfermedades, factores de riesgo, carencias e inequidades sociales que se reportan en varias regiones del país. El interés y la movilización política se fundamentan en la Constitución Política de Colombia, donde se definen los principales deberes y derechos de la población para la puesta en marcha de un diseño de intervenciones interinstitucionales e intersectoriales que mejoren la atención integral.

Cabe señalar que la política pública de infancia y adolescencia para el distrito capital surge a través de los planes de desarrollo de los gobiernos, adelantados en el periodo que va de 2004 a 2008 y denominados Política por la calidad de vida de niños, niñas y adolescentes "quiéreme bien, quiéreme hoy", que se mantuvo en el periodo siguiente (2008-2012) con el programa de gobierno "Bogotá positiva: para vivir mejor” (Alcaldía Mayor de Bogotá, 2011), los cuales trascienden en el periodo de gobierno actual y se materializan en una política pública distrital, sostenible en la agenda pública durante el tiempo.

En efecto, la intencionalidad del distrito para posicionar la política pública como mecanismo garante de calidad de vida logra, para el año 2004, contar con 53 políticas y planes de acción en los territorios de Bogotá; en el ámbito de salud, con un total de quince. Esto pone 
en evidencia que la iniciativa de control generó miradas individuales, lineales y diferenciadoras de los problemas priorizados en los territorios, los cuales anulan y excluyen a la mayor parte de la población, olvidando la complejidad y dinámica de la vida (Espinosa, 2015).

Como resultado, la población infantil se transforma en el curso de vida transversal para el desarrollo de estas políticas que se armoniza con las problemáticas distritales, nacionales y mundiales, que posicionan al niño, niña y adolescente en el centro de la gestión gubernamental bajo el marco garante de los derechos de los niños declarados en el mundo desde el siglo XX. A su vez, los derechos se transforman en el fundamento de un sistema político social basado en la promoción y garantía del desarrollo de personas (Fondo de las Naciones Unidas para la Infancia, 2007). Los derechos humanos que incluyen los derechos de los niños son concebidos como el contenido sustancial del sistema democrático y enmarcan el Estado y las familias en la obligación de respetar, proteger y garantizar los derechos económicos, políticos, sociales y culturales por medio de políticas y recursos que logren su satisfacción y libertad humana en los contextos donde se desarrollan: la familia, la escuela, la comunidad, las instituciones, el sistema religioso y cultural (Colombia, 2013; Pautassu \& Royo, 2012; Fondo de las Naciones Unidas para la Infancia, 2007).

El país ha invertido recursos en el desarrollo de iniciativas nacionales que impulsan la ejecución de acciones desde el distrito para mejorar las condiciones de vida del niño, niña y adolescente. Lo anterior es viable y legítimo mediante el compromiso interinstitucional enmarcado en las experiencias previas de los gobiernos que, a través de políticas como "Quiéreme bien, quiéreme hoy" (2004-2008) y "Por la calidad de vida de niños, niñas y adolescentes" (2008-2010), proporcionaron ejes de diseño, implementación y evaluación claves en la actualización y en la reformulación de la política emitida para 2011 (Alcaldía Mayor de Bogotá, 2011).

A pesar de las acciones ejecutadas bajo este marco político, estas no han logrado producir cambios en la realidad de la ciudad y se consideran elementos que han dificultado el éxito de la política en Colombia por varias 
razones identificadas: 1) no se vinculan con la realidad del territorio que se caracteriza por ser susceptible a eventos impredecibles, intersubjetivos, caóticos y dinámicos, que requieren ser nutridos desde la construcción social bajo procesos de interacción y comunicación con los sujetos; dicha construcción está cargada de subjetividad, con patrones de organización similares pero simultáneamente diferentes (Ordoñez-Matamoros, 2013); 2) baja participación de los niños y adolescentes en las distintas etapas de la política pública distrital; y 3) carencia de compromiso institucional por la continuidad de las intervenciones y la disponibilidad de los recursos suficientes para su sostenibilidad (Simarra, 2010).

En este sentido, la carencia de investigaciones sobre políticas públicas, junto con las debilidades metodológicas y conceptuales identificadas en la puesta en marcha generan preocupación en las autoras por la pérdida progresiva del valor social que tiene la política pública en el desarrollo colectivo, así como por la generación de desconfianza y deslegitimización de las acciones gubernamentales y su efectividad en la calidad de vida y desarrollo del niño, niña y adolescente.

\section{Metodología}

Se realizó un análisis cualitativo de base documental fundamentado en la metodología propuesta por Ordoñez-Matamoros (2013), la cual pretende contribuir a una mayor comprensión del análisis de las políticas públicas, teniendo en cuenta las fases presentadas por el autor: tipología, identificación del problema, planeación, implementación, evaluación y reconocimiento de actores. La revisión documental consistió en la búsqueda, análisis y comprensión de hallazgos relevantes, identificados en artículos publicados en revistas indexadas y en literatura gris con base en los criterios metodológicos establecidos por el autor mencionado. Lo anterior facilitó la identificación de aspectos clave para cada una de las etapas de la política que se utilizan para este ejercicio académico. 


\section{Resultados}

\section{Tipología}

La Política Pública de Infancia y Adolescencia en Bogotá, D. C., 20112021 es de tipo mixta. Es distributiva porque el modo en que están descritos el contenido, los ejes y los componentes infieren la asignación de recursos a un grupo social específico, en este caso, los niños, niñas y adolescentes. Esto otorga beneficios económicos, sociales, políticos y culturales a la población de interés a mediano y largo plazo.

También se observa que dicha política está catalogada como regulativa por el intento que hacen los constructores de la misma de controlar acciones inmersas en el contexto que vulneran el desarrollo de la población y conllevan a la definición de parágrafos específicos de procesos judiciales y coercitivos: violencia, consumo y expendio de sustancias psicoactivas, explotación sexual y comercial, conflicto armado y problemas relacionados con hurtos y/u homicidios (Arroyave Alzate, 2010).

De igual forma, la política muestra matices de tipo redistributivo porque asigna los servicios de salud por ejes de prioridad de acuerdo con el ciclo vital y la exposición a situaciones intolerables que inciden en la calidad de vida y desarrollo de los niños, niñas y adolescentes: muertes por causas evitables, hambre y desnutrición, maltrato y/o abuso sexual, ausencia de cuidadores, exclusión social por etnia, religión y/o discapacidad (Alcaldía Mayor de Bogotá, 2011).

\section{Identificación del problema}

Una de las etapas más complejas de análisis de la política pública es la identificación del problema debido a que si este no está bien identificado, se corre el riesgo de no acertar al momento de proponer soluciones. Lo que hace reiterativo que la definición del problema sea vital para definir el curso de las acciones o alternativas de solución (Ordoñez-Matamoros, 2013). 
Como producto de este ejercicio, en la política de infancia y adolescencia en Bogotá, D. C. 2011-2021, se evidencian algunos ajustes estructurales realizados a la luz de cambios políticos e institucionales, lo que llevó a que la problemática identificada en la primera versión haya tenido una serie de transformaciones para la versión final. Una de ellas fue el aumento del número de problemas y situaciones definidas como prioritarias. Para la política actual, se tuvieron en cuenta los siguientes aspectos para la definición de los problemas en el distrito, tal como se menciona en la tabla 1 (Identificación de problemas política pública de infancia y adolescencia en Bogotá, D. C., 2011-2021).

\section{Tabla 1. Identificación de problemas política pública de infancia y adolescencia en Bogotá, D. C., 2011-2021}

\begin{tabular}{|c|c|}
\hline Análisis inicial & Análisis actual \\
\hline $\begin{array}{l}\text { Un horizonte de sentido } \\
\text { para las acciones del distrito } \\
\text { capital, dirigidas a la mejora } \\
\text { de la calidad de vida de niños, } \\
\text { niñas y adolescentes el cual } \\
\text { establece que: "La calidad de } \\
\text { vida es la satisfacción de los } \\
\text { intereses y las necesidades } \\
\text { sociales, materiales y afectivas } \\
\text { de las niñas y los niños; estas } \\
\text { necesidades e intereses se } \\
\text { derivan de sus derechos, cuyo } \\
\text { ejercicio pleno es el horizonte } \\
\text { al que hay que tender". }\end{array}$ & $\begin{array}{l}\text { La protección integral para la garantía de los } \\
\text { derechos de niños y niñas tal como está expuesta } \\
\text { en la Ley } 1098 \text { de } 2006 \text {, debe ser introducida como } \\
\text { enfoque clave y ser visibilizada en la estructura de } \\
\text { la política. }\end{array}$ \\
\hline $\begin{array}{l}\text { Seis situaciones inadmisibles } \\
\text { que constituían "lo que la } \\
\text { población podría reconocer } \\
\text { fácilmente como asuntos } \\
\text { que no se pueden tolerar en } \\
\text { la ciudad" en relación con la } \\
\text { infancia y la adolescencia. }\end{array}$ & $\begin{array}{l}\text { Los ejes generadores de acción, tal como estaban } \\
\text { formulados, han cumplido ya su función de } \\
\text { abordar "temas fuertemente convocantes" } \\
\text { en torno a la garantía de los derechos de los } \\
\text { niños, niñas y adolescentes y deben transformar } \\
\text { su intencionalidad para convertirse en ejes } \\
\text { programáticos o estratégicos de la política en } \\
\text { relación con el desarrollo de los niños, las niñas y } \\
\text { los adolescentes. }\end{array}$ \\
\hline
\end{tabular}




\begin{tabular}{|c|c|}
\hline Análisis inicial & Análisis actual \\
\hline $\begin{array}{l}\text { Once ejes de identidad que } \\
\text { daban forma y sentido a } \\
\text { la finalidad de la política, } \\
\text { redactados a la manera de un } \\
\text { sueño. }\end{array}$ & $\begin{array}{l}\text { Los ejes generadores de acción, tal como estaban } \\
\text { formulados, han cumplido ya su función de abordar } \\
\text { "temas fuertemente convocantes" en torno a la garantía } \\
\text { de los derechos de niños y niñas y deben transformar su } \\
\text { intencionalidad para convertirse en ejes programáticos } \\
\text { o estratégicos de la política en relación con el desarrollo } \\
\text { de los niños, las niñas y los adolescentes. }\end{array}$ \\
\hline \multirow[t]{3}{*}{$\begin{array}{l}\text { Tres ejes generadores de acción } \\
\text { "sobre temas fuertemente } \\
\text { convocantes de todos los } \\
\text { sectores y actores sociales, } \\
\text { que al abordarlos permitieran } \\
\text { incidir en muchos de los } \\
\text { factores determinantes de la } \\
\text { calidad de vida de los niños, las } \\
\text { niñas y los adolescentes" con } \\
\text { sus componentes y líneas de } \\
\text { acción. }\end{array}$} & $\begin{array}{l}\text { En particular los ejes generadores de acción, en } \\
\text { su transformación a ejes programáticos, deben } \\
\text { organizar las acciones intencionadas de Bogotá } \\
\text { con enfoque diferencial que considere todas las } \\
\text { etapas del ciclo vital, desde la gestación hasta } \\
\text { la adolescencia, de acuerdo con los temas y las } \\
\text { situaciones relevantes para la ciudad, identificados } \\
\text { por los diagnósticos. }\end{array}$ \\
\hline & $\begin{array}{l}\text { Los componentes actualizados de la política pública } \\
\text { de infancia y adolescencia de Bogotá, D. C. para los } \\
\text { siguientes diez años deben considerar los temas y } \\
\text { las situaciones que, en su trayectoria, demostraron } \\
\text { ser significativos por su impacto en la calidad de } \\
\text { vida de niños, niñas y adolescentes y relevantes } \\
\text { en la promoción, prevención, garantía, ejercicio y } \\
\text { restablecimiento de los derechos de niños, niñas y } \\
\text { adolescentes en la ciudad, en particular aquellos que } \\
\text { no fueron introducidos en la formulación de } 2004 \text {. }\end{array}$ \\
\hline & $\begin{array}{l}\text { Acorde con el enfoque diferencial y con la } \\
\text { protección integral de sus derechos, los grupos } \\
\text { de derechos al desarrollo y a la ciudadanía en } \\
\text { particular, los derechos a la participación, a } \\
\text { la cultura, al patrimonio, al arte, al juego, a la } \\
\text { recreación y al deporte deben ser visibilizados } \\
\text { por referencia a su relación con la formación del } \\
\text { niño, la niña y los adolescentes como ciudadanos } \\
\text { y ciudadanas y sujetos políticos en Bogotá, D. C. } \\
\text { Esto en respuesta a los retos que representarán } \\
\text { para ellos y ellas los nuevos contextos de ciudad } \\
\text { que se prevén para los próximos diez años. }\end{array}$ \\
\hline
\end{tabular}




\begin{tabular}{|l|l|}
\hline \multicolumn{1}{|c|}{ Análisis inicial } & \multicolumn{1}{|c|}{ Análisis actual } \\
\hline & $\begin{array}{l}\text { Las familias y los cuidadores y cuidadoras de niños } \\
\text { y niñas, desde la gestación hasta la adolescencia, } \\
\text { deben considerarse en esta política como } \\
\text { corresponsables en la garantía de sus derechos. } \\
\text { Por tanto, las capacidades de las familias y los } \\
\text { cuidadores y cuidadoras para el cumplimiento de } \\
\text { ese rol deben ser promovidos y fortalecidos desde } \\
\text { las acciones de la misma. }\end{array}$ \\
\hline $\begin{array}{l}\text { La revisión de la formulación de la política pública } \\
\text { de infancia y adolescencia de Bogotá, D. C. debe } \\
\text { responder a una formalización que provea las } \\
\text { condiciones para que, en lo sucesivo, se diseñe un } \\
\text { plan de acción para su implementación y se realicen } \\
\text { procesos continuos de seguimiento, monitoreo y } \\
\text { evaluación del mismo a partir de una arquitectura } \\
\text { institucional y de indicadores claramente definidos. }\end{array}$ \\
\hline
\end{tabular}

Fuente: Elaboración propia.

$\mathrm{El}$ anterior esquema conlleva a inferir que es importante reconocer el contexto de la política pública, lo que justifica que los problemas no son algo concreto, no se derivan de constructos mentales; se interpretan de forma estratégica a conveniencia del tomador de decisión y de la estructura de gobierno de la política. En este sentido, el proceso analítico se debe abordar desde todas las fases de la política pública con el fin de no caer en errores como, por ejemplo, del tercer tipo, el cual explica claramente la toma de decisión frente a un problema equivocado (Ordoñez-Matamoros, 2013), es decir, “[...] ofrecer una solución al problema que no es y que puede generarse por varias razones, entre ellas: 1) no se reconoce que el problema hace parte de un sistema de problemas; 2) no se reconoce que existen jerarquías de problemas; y 3) no se reconoce que cada elemento constitutivo del problema ejerce un efecto sobre los demás” (Montenegro Hidalgo, 2015).

Por otro lado, los problemas definidos en las políticas públicas también son necesidades, valores y oportunidades de desarrollo, que no están resueltos y que son posibles de alcanzar mediante la acción pública. Dichos problemas no son independientes, por lo general hacen parte de un sistema complejo. Pero el enorme reto de la definición del pro- 
blema es no confundirlo con situaciones problemáticas, ya que estas últimas son creadas por el ser humano mediante la interpretación y el juicio. Lo anterior va en contravía del deber ser de la definición del problema, percibido por la sociedad que define el problema y las posibles alternativas de solución y no por las instituciones que desconocen las realidades locales.

Cabe resaltar que, en la política pública de infancia y adolescencia del distrito, se observa que los problemas están mal estructurados porque: 1) son definidos por muchos actores; y 2) hay un amplio panorama de alternativas planteadas que no se conocen con precisión, así como la probabilidad de ocurrencia y aplicabilidad en el territorio.

La infancia es una variable de análisis social. Por lo tanto, no puede nunca ser analizada de manera individual y ajena a otras variables como la clase social, el género o la pertenencia étnica. Un análisis comparativo y multicultural revela una variedad de infancias más que un fenómeno singular y universal (Jofré, 2007).

\section{Planeación}

La política de infancia y adolescencia plantea la definición de criterios como estructura clave para la elección de las alternativas de solución. Dichos parámetros giran en torno a la protección integral de la población objetivo por medio del abordaje por ciclo vital, desde la gestación hasta la adolescencia, con enfoques diferenciales de etnia, condiciones de vulnerabilidad y discapacidad, así como redes de apoyo social (Alcaldía Mayor de Bogotá, 2011) bajo marcos normativos, ético-políticos, conceptuales y analíticos.

Dentro de esta política, los actores de decisión y expertos en el tema establecen principios, prioridades, metas y objetivos a lograr, coherentes con el propósito definido en la política: "[...] asegurar la realización de los derechos individuales y colectivos para que desarrollen sus capacidades y potencialidades" (Alcaldía Mayor de Bogotá, 2011), p. 2. Dichos elementos son el resultado de decisiones derivadas de lucha entre intereses materiales e ideológicos que implican mecanismos 
de formulación y legitimación de las alternativas de solución que, en la política, se describen claramente.

Es notable la ausencia del reconocimiento del contexto territorial desde la subjetividad de los individuos que a diario se enfrentan a eventos que pueden ser desconocidos desde una mirada externa. Dicha mirada está inmersa en procesos de racionalidad individual que, al ser sumada con la de otros individuos, consolida la elección racional del colectivo, tal como se observa en el documento. Así mismo, se identifica un incrementalismo desarticulado porque no hay una revisión profunda de líneas de base desarrolladas en el pasado que orienten las decisiones actuales y facilitan la no repetición de errores y fracasos (Ordoñez-Matamoros, 2013).

De igual forma, es visible en la política la falta de indagación por la proyección de consecuencias derivadas de las soluciones analizadas, lo que impide diferenciar los métodos aplicados para la selección y rechazo de las alternativas, teniendo en cuenta que dichos métodos son el resultado de un ejercicio cuantitativo dado en espacios de discusión, negociación y comunicación (Ordoñez-Matamoros, 2013).

Lo anterior no se observa esquematizado en matrices, cuadros o herramientas que describan las variables a analizar y los actores a intervenir comparándolos con las alternativas de solución a estudiar. Este ejercicio posibilita la definición de los criterios de elección para la alternativa más conveniente, de forma eficaz, eficiente y organizada, alejándose de la cultura de toma decisiones a priori como tal vez se infiere que sucedió en esta política (Ordoñez-Matamoros, 2013).

El último mecanismo implícito en la formulación de la política es la legitimación de la nueva decisión por actores particulares, considerados clave en las siguientes fases de la política pública. Este proceso solo es visible en la firma del tomador de decisión: la alcaldesa encargada de la ciudad, por medio de la reglamentación de la política como decreto que le atribuye particularidades de obligatorio cumplimiento; de esta manera, se transforma en una norma oficial revestida de autoridad y símbolo ante el Estado, pero no ante la población objeto (Roth, 2002). 
Luego de realizar el análisis y legitimación de las decisiones tomadas y argumentadas, se procede al diseño de soluciones a través de diversas técnicas que precisan los fines, objetivos y estrategias de ejecución de forma secuencial y jerárquica, lo que coincide con las descripciones que se realizan en la política pública que se está analizando. Lo anterior se sintetiza en la tabla 2 (Características de la Política Pública de Infancia y Adolescencia en Bogotá, D. C., 2011-2021).

Tabla 2. Características de la Política Pública de Infancia y Adolescencia en Bogotá, D. C., 2011-2021

\begin{tabular}{|c|c|c|}
\hline \multicolumn{3}{|c|}{ Política Pública de Infancia y Adolescencia en Bogotá, D. C., 2011-2021 } \\
\hline Propósito & Principios & Actores \\
\hline \multicolumn{3}{|c|}{ Definición de situaciones intolerables } \\
\hline $\begin{array}{c}\text { Eje No. 1: niños, niñas y } \\
\text { adolescentes en ciudadanía } \\
\text { plena }\end{array}$ & $\begin{array}{c}\text { Eje No. 2: Bogotá } \\
\text { construye ciudad con } \\
\text { los niños, las niñas y los } \\
\text { adolescentes }\end{array}$ & $\begin{array}{l}\text { Eje No. 3: gobernanza por } \\
\text { la calidad de vida de la } \\
\text { infancia y la adolescencia }\end{array}$ \\
\hline Componentes & Componentes & Componentes \\
\hline $\begin{array}{l}\text { Ciudad, familias y } \\
\text { ambientes seguros. }\end{array}$ & $\begin{array}{l}\text { Escenarios para la } \\
\text { ciudadanía. }\end{array}$ & $\begin{array}{l}\text { Acciones intencionadas y } \\
\text { diferenciales de protección } \\
\text { de los niños y las niñas } \\
\text { desde la primera infancia } \\
\text { hasta la adolescencia } \\
\text { gestionadas de manera } \\
\text { integral. }\end{array}$ \\
\hline Alimentación nutritiva. & Movilización social. & $\begin{array}{l}\text { Sociedad civil, personas } \\
\text { y organizaciones de la } \\
\text { ciudad corresponsables de } \\
\text { la garantía de los derechos } \\
\text { de los niños, las niñas y } \\
\text { los/las adolescentes. }\end{array}$ \\
\hline Creciendo saludables. & $\begin{array}{l}\text { Redes de cuidado } \\
\text { calificado de niños, niñas } \\
\text { y adolescentes desde la } \\
\text { primera infancia. }\end{array}$ & $\begin{array}{l}\text { Las familias con vínculos } \\
\text { seguros, corresponsables } \\
\text { en la generación de } \\
\text { condiciones para el } \\
\text { desarrollo de los niños, } \\
\text { las niñas y los/las } \\
\text { adolescentes. }\end{array}$ \\
\hline
\end{tabular}




\begin{tabular}{|c|c|c|}
\hline $\begin{array}{l}\text { Igualdad de oportunidades } \\
\text { para vivir la identidad de } \\
\text { género para niños y niñas } \\
\text { desde la primera infancia. }\end{array}$ & $\begin{array}{l}\text { Interacción y diálogo } \\
\text { intergeneracionales en } \\
\text { condiciones de equidad. }\end{array}$ & \\
\hline $\begin{array}{l}\text { Sexualidad y re-creación } \\
\text { de la vida. }\end{array}$ & $\begin{array}{l}\text { Promoción y difusión } \\
\text { de lenguajes y estéticas } \\
\text { infantiles y adolescentes } \\
\text { sobre la ciudad en el } \\
\text { espacio público. }\end{array}$ & \\
\hline Felices de ser quienes son. & & \\
\hline $\begin{array}{l}\text { Educación para disfrutar y } \\
\text { aprender desde la primera } \\
\text { infancia. }\end{array}$ & & \\
\hline $\begin{array}{l}\text { Expresión auténtica desde } \\
\text { el disfrute del patrimonio } \\
\text { cultural, el arte, el juego, la } \\
\text { recreación y el deporte. }\end{array}$ & & \\
\hline $\begin{array}{l}\text { Participación con } \\
\text { incidencia. }\end{array}$ & & \\
\hline \multicolumn{3}{|c|}{ Indicadores } \\
\hline Categoría & Objetivos de la política & $\begin{array}{l}\text { Número de indicadores } \\
\text { definidos }\end{array}$ \\
\hline \multirow{4}{*}{ Existencia } & Todos vivos. & 5 \\
\hline & Ninguno desnutrido. & 5 \\
\hline & Todos saludables. & 20 \\
\hline & Ninguno sin familia. & 3 \\
\hline \multirow{3}{*}{ Desarrollo } & Ninguno sin educación. & 11 \\
\hline & Todos jugando. & 3 \\
\hline & $\begin{array}{l}\text { Todos capaces de manejar } \\
\text { afectos, emociones y } \\
\text { sexualidad. }\end{array}$ & 1 \\
\hline \multirow[t]{2}{*}{ Ciudadanía } & $\begin{array}{l}\text { Todos participando en } \\
\text { espacios sociales. }\end{array}$ & 3 \\
\hline & Ninguno sin registro. & 1 \\
\hline
\end{tabular}




\begin{tabular}{|c|l|c|}
\hline \multicolumn{2}{|c|}{ Indicadores } \\
\hline Categoría & \multicolumn{1}{|c|}{ Objetivos de la política } & $\begin{array}{c}\text { Número de indicadores } \\
\text { definidos }\end{array}$ \\
\hline \multirow{5}{*}{ Protección } & $\begin{array}{l}\text { Ninguno maltratado, } \\
\text { abusado o víctima del } \\
\text { conflicto interno generado } \\
\text { por grupos al margen de } \\
\text { la ley. }\end{array}$ & 7 \\
\cline { 2 - 4 } & $\begin{array}{l}\text { Ninguno en actividad } \\
\text { perjudicial. }\end{array}$ & 3 \\
\cline { 2 - 4 } & $\begin{array}{l}\text { Adolescentes acusados de } \\
\text { violar la ley penal con su } \\
\text { debido proceso. }\end{array}$ & 3 \\
\hline Financieros & Gasto público. & 14 \\
\hline
\end{tabular}

Fuente: Elaboración propia.

La tabla 2 muestra que la formulación de soluciones coincide con el método de cascadas de metas y objetivos que delimita los niveles de intervención, así como la atribución de los recursos disponibles para la ejecución. Adicionalmente, los indicadores definidos son parte del método de efectos e indicadores que complementan el esfuerzo planificador de los tomadores de decisión (Roth, 2002).

\section{Implementación}

El proceso de implementación de la política pública parte de la puesta en marcha del Decreto 520 de 2011, en el cual se menciona que resulta absolutamente necesario que la ciudad adopte formalmente la Política Pública de Infancia y Adolescencia en Bogotá, D. C., 2011-2021, porque se garantiza un avance progresivo y sistemático en la defensa y protección integral de los niños, las niñas y los/las adolescentes de la ciudad.

De igual forma, se hace mención a que la administración distrital actuará a través de su organización administrativa de manera armónica, mediante la articulación de programas, proyectos y acciones administrativas a nivel interinstitucional, sectorial, intersectorial y 
transectorial, para lograr la eficiencia y economía de la gestión pública, con participación de los actores gubernamentales.

Una de las fases esenciales en el ciclo de una política pública es su implementación. En la actualidad, es evidente que no es tan fácil debido a la fragmentación de acciones para la puesta en marcha; en este caso el modelo de implementación que se utilizó es top-down, de arriba hacia abajo, mencionado en el mismo decreto y asignado por funciones a la Secretaría Distrital de Integración Social. Se le asignó, entre otras actividades, la función de formular, orientar y desarrollar políticas sociales en coordinación con otros sectores, organismos o entidades, para los distintos grupos poblacionales, familias y comunidades, en especial aquellos en mayor situación de pobreza y vulnerabilidad, y promover estrategias que permitan el desarrollo de sus capacidades.

Esta concepción de implementación basada en que la administración pública es el actor ideal y el primero en desplegar las funciones y acciones al territorio, conlleva posiblemente a la participación pasiva y casi nula de los actores sociales. Se evidencia una clara asignación de tareas y responsabilidades a las secretarías distritales para llevar a cabo el desarrollo de dicha política a través del plan de acción de la Política Pública de Infancia y Adolescencia en Bogotá, D. C., 20112021, inmerso dentro de las metas del plan de desarrollo para la ciudad.

\section{Evaluación}

La evaluación de las políticas públicas es una herramienta técnicopolítica que permite visualizar los resultados de las decisiones tomadas e implementadas en un tiempo determinado; se realiza a través de la recolección, verificación e interpretación de la información (Muller, 2002). Los mecanismos evaluativos se definen en la etapa de formulación; en esta política se establecieron aproximadamente 79 indicadores a través de un abordaje netamente cuantitativo.

Esta fase se puede desarrollar de forma auto y hetero-evaluativa como estrategia reflexiva y de debate entre los actores implicados que 
calificarán los recursos y las capacidades sociales para el desarrollo de acciones en los colectivos.

A partir de los procesos evaluativos, las estructuras de gobierno comprenden, de forma profunda, los procesos sociales inmersos en la política pública y el real fundamento de las acciones. De igual forma, esta es una fase que permite la comunicación, en escenarios públicos, de la rendición de cuentas y del balance general de los objetivos alcanzados, pues se considera que este es un canal de retroalimentación para la ciudadanía, establecido como un acto administrativo de la política pública, que también tiene como ventaja el control de los recursos de acuerdo con las responsabilidades otorgadas.

Dentro de los momentos de evaluación definidos está el ex ante, o a priori, como mecanismo de cálculo de factibilidad y de los efectos generados por la implementación de la política. No se ha registrado este ejercicio en el documento analizado ya que no hay uso de métodos cuantitativos que faciliten la proyección de las consecuencias de las alternativas de solución seleccionadas.

La evaluación concomitante, que se realiza de forma simultánea a la implementación de la política pública, con el objeto de monitorear los avances y dificultades en el proceso, se define dentro de la política como el sistema de monitoreo de las condiciones de vida de la infancia y adolescencia, constituido bajo procesos normativos que obligan al cumplimiento de avances por parte de la Secretaría de Integración Social, con apoyo interinstitucional. Dicho sistema de información vigila los logros que se observan en términos de calidad de vida en los componentes definidos en la política y de acuerdo con las acciones implementadas. Los resultados son socializados anualmente a todos los actores y sectores involucrados en la atención de la población objeto (Jaramillo, 2007).

Por otra parte, de la evaluación expost, o a posteriori, está orientada hacia el análisis de los efectos de la política pública luego de la implementación, como mecanismo de aprendizaje, para las decisiones futuras (Muller, 2002). En la política pública de infancia y adolescencia se define a través de los indicadores de evaluación que, al ser calculados, 
proyectarán los logros obtenidos luego de la ejecución y serán socializados en la rendición de cuentas que se programa anualmente (Alcaldía Mayor de Bogotá, 2011).

\section{Reconocimiento de actores}

La movilización de actores por la primera infancia tuvo origen en la "Alianza por la Política Pública de Infancia y Adolescencia en Colombia", a través de un grupo de trabajo integrado por el Instituto Colombiano de Bienestar Familiar (ICBF), el Departamento Administrativo de Bienestar Social, el Centro Internacional de Educación y Desarrollo (CINDE), Save the Children y UNICEF, que fundamentaron las líneas técnicas para la elaboración e implementación de políticas en los departamentos y municipios del país.

Hay que mencionar, además, que en el ámbito distrital los actores que participaron en la construcción e implementación de la Política Pública de Infancia y Adolescencia en Bogotá, D. C., 2011-2021 hicieron parte del Comité Operativo Distrital de Infancia y Adolescencia en el marco de lo establecido por el Consejo Distrital de Política Social. Dicho comité contó con el apoyo de la Subdirección para la Infancia de la Secretaría Distrital de Integración Social, el equipo de análisis y seguimiento de la política de infancia y adolescencia y con la asesoría en política pública de Save the Children, bajo el marco del convenio de impulso a la política y primera infancia.

\section{Discusión}

La política pública está compuesta por acciones gubernamentales que median las relaciones de los sectores y modifican sus relaciones; es una forma de potencializar la gestión de la relación global-sectorial (Muller, 2002). El diseño de la política pública para este ejercicio fue de tipo mixta — regulativa de tipo redistributiva-, lo cual responde a problemáticas o necesidades sociales, pero de acuerdo con las experiencias desarrolladas se muestra que no solo debe llevarse a cabo para modificar los riesgos atribuibles a la salud enfocados en la enfermedad, sino también debe incluir a la población sana. 
Específicamente, en la valoración de la etapa de identificación del problema, se observó que las necesidades definidas fueron interpretadas a conveniencia por el tomador de decisión y de acuerdo con la estructura de gobierno de la política, junto con una debilidad en la estructuración en donde la causa atribuible fue la cantidad de personas que toman decisiones que, por lo general, tienen intereses particulares. Dichas decisiones presentan varias alternativas que, usualmente, son posibilidades inciertas debido al accionar fragmentado de las instituciones.

Por otra parte, los problemas se formulan, "[...] como la ausencia de una solución o la falta de un determinado bien o servicio, que conduciría a elaborar respuestas que solo se orientan a proveer lo que falta, cuando esta carencia es el efecto de causas más profundas que constituyen la verdadera naturaleza del problema y que quedarían eclipsadas por la inadecuada definición del mismo" (Lucca \& Tecco, 2011 p. 107).

En este orden de ideas, los formuladores del problema partieron de la percepción del mismo sin hacer un análisis estructural junto con los demás factores influyentes que podrían tener relación con la situación problema, es decir, lo que se llama definición del metaproblema (Lucca \& Tecco, 2011).

Esta mirada integradora da paso a la definición del problema básico y general: si es de carácter económico, político o social, lo que condiciona la formulación final del problema desde miradas reflexivas a la interacción y a la dinámica del mismo, así como a las alternativas de solución disponibles (Lucca \& Tecco, 2011).

El panorama descrito anteriormente reitera lo que la literatura reporta con relación a la participación de los menores de edad en los asuntos políticos y que ha sido declarada una necesidad apremiante desde los organismos internacionales, como Naciones Unidas, a partir del año de 1998, ya que resulta una estrategia de reducción de errores en la formulación, análisis y comprensión formal de los problemas. 
Como resultado, los países están desarrollando espacios de debate y de reflexión pública sobre temas de interés de dicha población al promover la conciencia política, desde edades tempranas, junto con habilidades para el pensamiento crítico. Sin embargo, por el poco conocimiento y experiencia que se percibe y que, se supone, tienen los menores, no se reconocen los contenidos discutidos y las opiniones que se dan, incumpliendo así con las declaraciones internacionales y con los derechos establecidos.

Una experiencia importante con respecto a lo anterior se reportó en Finlandia, país que logra la participación activa y real de la población infantil y adolescente en estamentos gubernamentales al ser parte de la toma de decisiones y de asesorías, además de acompañar la gestión de las acciones intersectoriales (Kallio \& Häkli, 2011).

Otro aspecto evaluado fue el método seleccionado, en esta política, para la estructura del problema, basado en el análisis causa-efecto y que pierde de vista la perspectiva integradora. Debido a este enfoque tradicional en las políticas públicas, el análisis causal es frecuente, tal como se observa en este caso. No obstante, el análisis y la comprensión de los factores relacionados con la situación problema se hizo desde la mirada institucional, sin tener en cuenta la percepción real y las vivencias de la sociedad, en especial de los niños, niñas y adolescentes.

Con respecto a la etapa de planeación, se identifica que la política pública abarca el método de la definición de criterios, lo que va en contravía de lo metodológico para el proceso de formulación de alternativas de solución (Roth, 2002). Este sugiere que se desarrolle a partir de estrategias analíticas del problema para argumentar y contrargumentar las elecciones que no se identifican a primera vista en esta política. En el marco analítico, los autores realizan una consolidación de aspectos relevantes desde lo cuantitativo, derivado de lo institucional, y fundamentado en aspectos que se consideran de impacto negativo para la calidad de vida: necesidades básicas, tipología familiar, capacidad adquisitiva, servicios públicos, condiciones de vulnerabilidad, seguridad alimentaria, factores causales de muerte materna, perinatal, neonatal y de infancia, 
servicios de salud y educativos, cultura, recreación y deporte, además de procesos de participación.

Es importante tener en cuenta que los mecanismos de planificación técnica disponibles han sido diseñados para el control de los recursos, el cumplimiento de los objetivos y la reducción de la incertidumbre del futuro por inconsistencias que no se hayan previsto. Lo anterior se incumple a la luz de esta política, debido a que existe un desconocimiento de la situación real de la población objeto ya que no hay descripciones de subjetividades que plasmen los ejercicios de reconocimiento del otro y de su contexto: valores, formas de autoorganización, percepciones e ideologías, lo que convierte la política en un problema práctico que no resuelve, de forma profunda, las necesidades de los individuos, y convierte en insuficientes los criterios de elección del problema y de las alternativas de solución (Majone, 2005).

En este sentido, es vital que la gestión de la política pública comience con la lectura real de las necesidades, acercándose a la cotidianidad de los seres humanos, implementando a su vez diversos instrumentos que apoyen el diseño, la planeación, la puesta en marcha y la evaluación de la misma sin perder la participación activa de la comunidad durante todas las fases (Carey, Crammond \& Keast, 2014).

Así mismo, no se identifica que las intervenciones planteadas por la política pública hayan tenido un marco orientador basado en la evidencia, a través de experiencias internacionales. Por ejemplo, en algunos se reporta un aumento considerable de casos de obesidad en la población infantil y adolescente como una contraposición al evento prevalente de desnutrición en países en vías de desarrollo (Schlickmann Fraineri, et al., 2011).

Varios estudios muestran que este evento se atribuye a una carga genética, seguida de un entorno caracterizado por un alto consumo de alimentos ricos en calorías y carbohidratos, porciones grandes y consumo frecuente, así como estilos de vida sedentarios. Frente a esto se identifican múltiples experiencias de países en donde las políticas públicas se han orientado a las restricciones de alimentos con grasas 
saturadas, así como a la promoción de los mismos en algún medio de comunicación, incitando a una alimentación "más segura". Sin embargo, se encuentran matices de conflictos de intereses económicos por parte de las multinacionales; de igual forma, estas medidas han aumentado la necesidad de la población de consumo de dichos alimentos. Esto conlleva a que la política deba tener un análisis que impulse cambios en los comportamientos individuales y en las condiciones sociales, económicas y políticas que son realmente los factores causales de dicho evento (Brownell, Schwartz, Puhl, Henderson \& Harris, 2009; Ebbeling, Pawlak \& Ludwig, 2002).

Un ejemplo son las acciones intersectoriales que diseñó la ciudad de Portland, en Oregon (Estados Unidos), a través de la política de gobierno denominada "Vida activa" al desarrollar intervenciones no directas sobre el individuo, sino sobre el contexto, como introducir parques, jardines, senderos para caminar en las comunidades. De igual forma, con el programa "Somerville" invitó a la concertación de los actores proveedores de alimentos y de salud para llegar a acuerdos saludables y de actividades que contribuyan al logro del objetivo. En ese sentido también se consideran importantes las competencias de liderazgo e innovación del profesional en salud pública, quien debe planificar e implementar los cambios en la comunidad, así como la gestión de alianzas que consoliden los programas en una línea de tiempo. De esta forma se garantizan entornos comunitarios adecuados para la vida activa y confortable (Gantner \& Olson, 2012).

En relación con el análisis del proceso de toma de decisiones, inmerso en la política, se infiere que los quienes toman las decisiones se inclinan por remediar los problemas prexistentes, tal vez no categorizados como tal en la realidad del territorio, con el propósito de no resolverlos por completo. El llamado es a fortalecer los roles de los tomadores de decisión, que se definen como el producto de un ejercicio de aprendizaje articulado desde las vivencias de los individuos, sumado a lo que percibe por la institucionalidad, partiendo de que todos los actores contribuyen a la comprensión real del fenómeno (Ordoñez-Matamoros, 2013). 
Se debe tener en cuenta que el análisis de múltiples perspectivas es muestra de ello, pues la lluvia de ideas es el principal encuentro colectivo y de reconocimiento del otro, donde todas las personas son fuente de conocimiento y proporcionan un mayor entendimiento sobre el problema (Tabaka, Eyler, Dodson \& Brownson, 2015).

Para la etapa de implementación se observó que las estrategias mencionadas en la política pública no se articulan con los avances tecnológicos que han permitido mayores y mejores mecanismos de comunicación y que, hoy en día, los adolescentes utilizan de manera frecuente. Así, las acciones e intervenciones que se diseñan en esta política pública deben aprovechar estas nuevas herramientas integradas a la cotidianidad de muchos niños, niñas y adolescentes de la ciudad. Esta es una estrategia de intervención llamativa para que la población se siente atraída por las acciones políticas (Barreto, Castro \& Leta, 2014).

A su vez, se deben considerar nuevas formas de ver al niño, niña y adolescente con diferentes paradigmas, inquietudes y situaciones que reconocen la complejidad de la vida (Jaramillo, 2007). Para dar un ejemplo, Chile, a través de la Política Pública de Protección Social ofrece un programa de intervención denominado "Chile crece contigo" basado en el acompañamiento continuo a la familia como principal estrategia de comunicación afectiva y toma de decisiones, y que está inmerso en procesos sociales y comunitarios que facilitan el desarrollo infantil (Arcos, et al., 2013).

De igual forma, la política pública, durante su implementación, debe reconocer e incluir a la familia, vista desde sus particularidades de estructura y funcionalidad, las cuales no se ajustan necesariamente a las tradiciones, por ejemplo, religiosas. Esto lleva a reflexionar acerca de que el niño y el adolescente están en un proceso simultáneo y altamente complejo de búsqueda de identidad o de identidades y de diferenciación, por lo que la familia puede ser un apoyo o un obstáculo para el desarrollo de las facultades físicas, mentales, morales, espirituales y sociales (Arcos, et al., 2013; Carvalho Monte, Rodríguez Sampaio, Rosa Filho \& Santana Barbosa, 2011). Se infiere que la 
construcción de una política pública debe estar centrada en la vida y no en la enfermedad, debe ser una política que posibilite la gestión de una formación integral, con capacidad de reconocer la diferencia y favorecer formas de vida diversas, alejadas de visiones mecanicistas y tradicionales (Martins, Costa \& Rossetti-Ferreira, 2010).

Es importante tener en cuenta que para la implementación de la política pública se utilizan mecanismos de tipo top-down, lo que tendrá como efecto la baja participación de la población objeto y de sus familias. Es necesario reconocer que el problema aparece desde la definición de la política pública, incluso desde la concepción de las problemáticas que la generan. Esto es causa de una débil concepción de la ciudadanía y de la participación social que se deja para espacios y momentos específicos, restringidos por la agenda de las instituciones.

Otro punto es la etapa de evaluación en la cual se observó ausencia de impacto a largo plazo; el único ejercicio que podría coincidir con este momento es el de las metas que el distrito y el país deben cumplir con relación a los Objetivos de Desarrollo del Milenio que, desde 2016, han sido denominados Objetivos de Desarrollo Sostenible, teniendo en cuenta la línea base registrada que puede ser útil para la proyección de acciones a implementar (Muller, 2002), presentadas en informes.

De esta forma, se describieron escasos métodos que la política pública contempla para la medición de resultados. Es importante tener en cuenta que, en la actualidad, en la literatura disponible acerca de la evaluación los autores recomiendan realizar mediciones también para los niveles de recursos, resultados, impactos, eficiencia y satisfacción, pues son categorías innovadoras que integran procesos de subjetividad en las percepciones de las familias, los niños, niñas y adolescentes, así como en la construcción de información confiable, válida y real que facilita la identificación de experiencias exitosas, fracasos, limitaciones, nuevos actores, clarificación de los problemas definidos y confrontación de las acciones con la realidad del territorio (Muller, 2002).

Otro rasgo de la evaluación de la política pública, en términos de efectividad y eficacia sobre la situación considerada problema, desde 
un abordaje integral, es el paso más importante para el entorno político porque es una experiencia real de los éxitos, debilidades, flexibilidades, resultados y barreras que se identifiquen durante la implementación y que pueden dar un marco orientador a países que tienen características socioeconómicas y políticas similares (Howard, Gordon \& Jones, 2014).

En vista de que las estructuras de gobierno observan el compromiso inherente a la acción política denominado "promoción de la salud", se describen experiencias internacionales para lograr la coordinación de los sectores gubernamentales con una alta probabilidad de que los resultados de la política pública logren los cambios esperados y la satisfacción de las necesidades de los individuos (Carey, Crammond \& Keast, 2014).

De esta forma, la política pública se dinamiza al perder concepciones institucionales rígidas y ambiguas en los resultados obtenidos, derivados de la inadecuada interpretación de los problemas. Se reitera su análisis para la construcción de un problema público y su posterior puesta en agenda, asunto crítico a tener en cuenta en la generación de la política pública. Por el hecho de que los problemas pueden ser simples o complejos dependiendo del número de tomadores de decisión, de alternativas, del grado de consenso entre los actores involucrados, de los resultados y posibilidades de éxito o fracaso, los problemas simples tienen grandes limitaciones, mientras que para los complejos, los procesos son incalculables (Ordoñez-Matamoros, 2013).

Así mismo, la fragmentación de las alternativas de solución, la toma de decisiones inadecuadas y la ausencia de procesos de evaluación transforman los mecanismos de argumentación en "cáscaras vacías" donde a corto, mediano o largo plazo estarán inmersos en procesos anquilosantes con pérdida del apoyo político, credibilidad intelectual y legitimidad (Majone, 2005).

En cuanto a la etapa de reconocimiento de actores, se identifica que la política pública no es coherente con las recomendaciones de expertos, que sugieren que todas las etapas de la política, en este caso de 
infancia y adolescencia, deben ser fruto de un trabajo participativo y democrático. En el caso del distrito se reconoce que hubo intervención de todos los sectores y actores involucrados con el desarrollo, promoción, defensa y garantía de los derechos de los niños, niñas y adolescentes, previa a los análisis de los principales problemas y recomendaciones identificadas por la población objeto en el marco de los encuentros ciudadanos realizados durante 2004, situación que se menciona en el Decreto 520 de 2011; pero en el documento de la política, no se describe como método de identificación y selección de alternativas propuestas por la población.

En este sentido, Ordoñez-Matamoros (2013) hace referencia a que los actores son los individuos, grupos y organizaciones que tienen un interés en el problema o en la solución del mismo, y cuya implementación incide en desarrollo social. Por esto es necesario establecer, de forma implícita, el fomento de oportunidades, redes sociales y definición de conflictos potenciales. Lo anterior coincide con el concepto de política pública que hace referencia a fenómenos complejos; sus elementos se interrelacionan y al agregarlos unos a otros se genera una realidad que va más allá de la simple sumatoria de sus partes. La frase que Morin cita de Pascal es muy ilustrativa en esta parte del texto: "No puedo concebir el todo sin concebir a la partes y no puedo concebir a las partes sin concebir al todo" (Morin, 2001).

Sin embargo, a pesar del reconocimiento del rol esencial de los actores en la política, en este caso de infancia y adolescencia del distrito la participación en las etapas de implementación y evaluación no es visible, lo que produce un sesgo legalista, que solo se focaliza en la toma de decisiones individuales y quizá limitadas.

\section{Reflexiones finales}

El proceso de identificación de tipologías de la política pública distrital permitió comprender la complejidad del ejercicio y entender que, en muchos casos, es válida la existencia implícita de múltiples tipos, dada la diversidad de los contenidos e intencionalidades enmarcados en ella (Roth, 2002). Sin embargo, es necesario que sea incluyente con 
la población en general para que así se transforme en una ventana de oportunidades para el desarrollo humano en todas sus dimensiones.

El desarrollo adecuado de la política pública está directamente relacionado con el equipo multidisciplinario con que se cuente; es vital que posean el conocimiento y la experiencia necesaria para el abordaje de múltiples situaciones bajo una coordinación interinstitucional que identifique la situación real de la población objeto e implemente estrategias acordes con el ciclo vital e intereses, para la resolución de las necesidades identificadas y para el fortalecimiento de la participación pública (Payne, 2000).

Es importante que los actores gubernamentales y tomadores de decisión utilicen las metodologías disponibles para la elaboración de políticas públicas, basadas en la evidencia científica disponible (Zardo \& Alex, 2014) y den cumplimiento al desarrollo de cada una de las etapas, con el propósito de visualizar y medir los resultados tangibles para los colectivos y tomadores de decisión.

\section{Conflictos de interés}

Las autoras no declaran ningún conflicto de interés.

\section{Referencias}

Alcaldía Mayor de Bogotá. (2011). Política Pública de Infancia y Adolescencia en Bogotá, D. C., 2011-2021. Bogotá: Comité Operativo Distrital de Infancia y Adolescencia-CODIA.

Arcos, E., Muñoz, L. A., Sánchez, X., Vollrath, A., Gazmuri, P. \& Baeza, M. (2013). Efectividad del Sistema de Protección Integral de la Infancia en madres y niños vulnerables. Rev. Latino-Am. Enfermagem, 1-9.

Argüello, O. (1991). Desarrollo económico, politicas sociales y de población. Santiago de Chile: CEPAL.

Arroyave Alzate, S. (2010). Las políticas públicas en Colombia. Insuficiencias y desafíos. Revista del Departamento de Ciencia Política, 95-111. 
Barreto, P., Castro, F. \& Leta, J. (2014). Health research networks on the web: an analysis of the Brazilian presence. Cadernos de Saúde Pública, 30(2), 369-378. doi: http://dx.doi.org/10.1590/0102-311X00136812

Brownell, K., Schwartz, M., Puhl, R., Henderson, K. \& Harris, J. (2009). The need for bold action to prevent adolescent obesity. Journal of Adolescent Health, 45(3), 8-17. doi: http:/ /dx.doi.org/10.1016/j.jadohealth.2009.03.004

Butelo, E. (2012). Notas sobre infancia y teoría: un enfoque latinoamericano. Salud colectiva. 8(3), 287-298. Recuperado de http://www.unla. edu.ar/saludcolectiva/revista24/v8n3a06.pdf

Carey, G., Crammond, B. \& Keast, R. (2014). Creating change in government to address the social determinants of health: how can efforts be improved? BMC Public Health, 14(1087), 1-11. doi: http://doi. org/10.1186/1471-2458-14-1087

Carvalho Monte, F. F., Rodrigues Sampaio, L., Rosa Filho, J. S. \& Santana Barbosa, L. (2011). Adolescentes autores de atos infacionais: psicología moral e legislação. Psicologia \&o Sociedade, 125-134.

CEPAL, Naciones Unidas, UNICEF, SECIB. (2001). Construir equidad desde la infancia y la adolescencia en Iberoamérica. Santiago de Chile: CEPAL, UNICEF, SECIB.

Cobb, R., Ross, J.-K. \& Ross, M. H. (1976). Agenda building as a comparative political process. The American Political Science Review, 70(1), 126-138. Recuperado de http://www.jstor.org/stable/1960328

Colombia. (2013). Derechos de la infancia y la adolescencia: Principales instrumentos y normas internacionales y nacionales. Bogotá: Presidencia de la República.

Duque Daza, J. (2013). Concepciones de la política y su incidencia en la gestión pública. Acerca de los fundamentos de las decisiones públicas. El Ágora U.S.B., 13(1), 191-206. Recuperado de http:/ / www.scielo.org. co/scielo.php?script $=$ sci_abstract\&pid $=$ S1657-80312013000100008

Ebbeling, C., Pawlak, D. \& Ludwig, D. (2002). Childhood obesity: publichealth crisis, common sense cure. The Lancet, 473-482. doi: https: / / doi.org/10.1016/S0140-6736(02)09678-2

Espinosa. (2015). Sintesis de una experiencia de análisis de diseño, implementación, monitoreo de políticas públicas. Bogota, D. C.: Alcaldía Mayor de Bogotá; Subsecretaría de Salud Pública, Dirección de Epidemiología y Análisis de Políticas Públicas de Salud Colectiva. 
Fondo de las Naciones Unidas para la Infancia. (2007). Justicia y derechos del niño. Santiago de Chile: Fondo de las Naciones Unidas para la Infancia.

Fox, D. (2002). The politics of policy development in public health notes on three stories. Journal of Public Health Management \& Practice, 8(1), 65-67.

Gantner, L. \& Olson, C. (2012). Evaluation of public health professionals' capacity to implement environmental changes supportive of healthy weight. Eval Program Plann, 407-416. doi: http://doi.org/10.1016/j. evalprogplan.2012.01.002

Hernández, R. (2006). Políticas de población en América Latina: Estudio de caso. La Habana: Centro de Estudios Demográficos, Universidad de La Habana.

Howard, S. J., Gordon, R. \& Jones, S. C. (2014). Australian alcohol policy 2001-2013 and implications for public health. BMC Public Health, 14(848), 2-13. doi: https://doi.org/10.1186/1471-2458-14-848

Jaramillo, L. (2007). Concepción de la infancia. Instituto de Estudios Superiores en Educación Universidad del Norte, 1-16.

Jofré, J. (2007). El recreo de la infancia: Argumentos para otros comienzos. Salud colectiva, 3(1), 213-214.

Kallio, K. P. \& Häkli, J. (2011). Tracing children's politics [in] political geography. Political Geography, 30(2), 99-109. doi: https://doi.org/10.1016/j. polgeo.2011.01.006

Lucca C., \& Tecco C. (2011). El proceso de estructuración de problemas: insumo para la formulación de políticas socio-habitacionales a escala local. Iberoamericana de Estudios Municipales, 105-131.

Majone, G. (2005). Evidencia, argumentacion y persuasion en la formulación de politicas públicas. Ciudad de México: Fondo de Cultura Económica.

Martins, L. B., Costa, N. R. \& Rossetti-Ferreira, M. C. (2010). Acolhimento familiar: caracterização de um programa. Paidéia (Ribeirão Preto), 20(47), 359-370. doi: http://dx.doi.org/10.1590/S0103-863X2010000300008

Milne, E. M. (2012). A public health perspective on transport policy priorities. Journal of Transport Geography, 21, 62-69. doi: https://doi. org/10.1016/j.jtrangeo.2012.01.013

Montenegro Hidalgo, V. (2015). Manual de análisis y diseño de políticas públicas, dirigido por Gonzalo Ordóñez-Matamoros (Reseñas). Quito: Flacso.

Morin, E. (2001). El paradigma de la complejidad: Introducción al pensamiento complejo. Barcelona: Española.

Muller, P. (2002). Las políticas públicas. Bogotá, D. C.: Universidad Externado de Colombia. 
Nisbetta, N., Gillespieb, S., Haddadb, L. \& Harrisb, J. (2014). Why worry about the politics of childhood undernutrition? World Development, 64, 420-433. doi: http://doi.org/10.1016/j.worlddev.2014.06.018

Ordoñez-Matamoros. (2013). Manual de análisis y diseño de políticas públicas. Bogotá, D. C.: Universidad Externado de Colombia.

Pautassu, L. \& Royo, L. (2012). Enfoque de derecho en las políticas de infancia: Indicadores para su medición. Santiago de Chile: CEPAL, Naciones Unidas. Payne, H. (2000). The health of children in public care. Caerphilly-Gales: University of Walles College of Medicine.

Regan, S., MacDonald, M., Allan, D. E., Martin, C. \& Peroff-Johnston, N. (2014). Public health human resources: A comparative analysis of policy documents in two Canadian provinces. Human Resources for Health, 12(13), 1-11. doi: https://doi.org/10.1186/1478-4491-12-13

Restrepo-Ochoa, D. A. (2013). La salud y la vida buena: Aportes del enfoque de las capacidades de Amartya Sen para el razonamiento ético en salud pública. Cad. Saúde Pública, 2371-2382. doi: http:/ / dx.doi. org/10.1590/0102-311X00069913

Roth, D. (2002). Políticas públicas: Formulación, implementación y evaluación. Bogotá, D. C.: Ediciones Aurora.

Salas-Zapata, W., Ríos-Osorio, L., Gómez-Arias, R. D. \& Castillo, X. Á.-D. (2012). Paradigmas en el análisis de políticas públicas de salud: limitaciones y desafíos. Revista Panamericana de Salud Pública, 77-81.

Sánchez, G. G. (2003). Gerencia pública y política social en Latinoamérica. Economía, Sociedad y Territorio, IV(13), 27-162.

Schlickmann FrainerI, D. E., Monteiro da Silva II, M. d., Portela de Santana II, M. L., Nedja Silva, d. S., Oliveira II, L. P., Barreto, M. L. \& Assis, A. M. (2011). Prevalence and associated factors of overweight in adolescents from Salvador, Bahia, Brazil. Rev Bras Med Esporte, 102-106. doi: http:// dx.doi.org/10.1590/S1517-86922011000200006

Simarra, J. (2010). Panorama de las políticas públicas de primera infancia en Colombia. Miscelánea Comillas, 68(132), 397-417.

Skelton, T. (2013). Children young people and politics: Transformative possibilities for a discipline? Geoforum. Elsevier, 4-6. doi: http://doi. org/10.1016/j.geoforum.2013.02.003

Tabaka, R. G., Eyler, A. A., Dodson, E. A. \& Brownson, R. C. (2015). Accessing evidence to inform public health policy: A study to enhance 
advocacy. Public Health, 129(6), 698-704. doi: http://doi.org/10.1016/j. puhe.2015.02.016

Tubbing, L., Harting, J. \& Stronks, K. (2015). Unravelling the concept of integrated public health policy: Concept mapping with Dutch experts from science, policy, and practice. Health Policy, 119(6), 749-759. doi: https://doi.org/10.1016/j.healthpol.2014.12.020

Valdés, J. \& Ospina, M. (2011). América Latina y el Caribe: La política social en el nuevo contexto. Montevideo: UNESCO.

Welti, C. (s. f.). Política de población y desarrollo económico. Ciudad de México: Instituto de Investigaciones Sociales, UNAM.

Zardo, P. \& Alex, C. (2014). Measuring use of research evidence in public health policy: A policy content analysis. BMC Public Health, 14(496), 2-10. doi: https://doi.org/10.1186/1471-2458-14-496 